
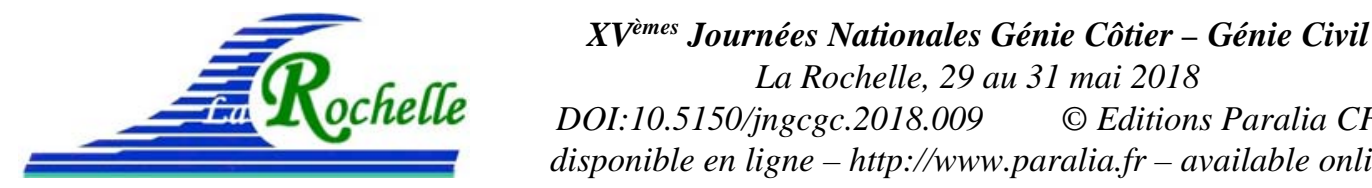

DOI:10.5150/jngcgc.2018.009 (C) Editions Paralia CFL

disponible en ligne - http://www.paralia.fr - available online

\title{
Effet de la remontée du niveau marin sur la marée : cas du plateau nord-ouest européen
}

\section{Déborah IDIER ${ }^{1}$, François PARIS ${ }^{1}$, Gonéri LE COZANNET ${ }^{1}$, Faiza BOULAHYA ${ }^{1}$, Franck DUMAS ${ }^{2}$}

1. BRGM, DRP/R3C,

3 avenue Claude Guillemin, 45060 Orléans, France.

d.idier@brgm.fr ; f.paris@brgm.fr;g.lecozannet@brgm.fr ; f.boulahya@brgm.fr

2. SHOM, Centre de Brest,

13, rue du Chatellier, 29228 Brest, France.

fdumas@shom.fr

\section{Résumé :}

L'élévation du niveau moyen des mers peut modifier non seulement les niveaux d'eau totaux, mais aussi la dynamique des marées. Cet article s'intéresse aux modifications induites sur les marées du plateau continental ouest-européen (en particulier sur le niveau de pleine-mer relatif), par une approche de modélisation. Des scénarios de remontée uniforme du niveau moyen sont considérés. Si les rivages actuels restent protégés de la submersion, alors, le niveau relatif de pleine-mer évolue proportionnellement avec la remontée du niveau marin sur la plus grande partie du plateau, tant que cette dernière reste inférieure à $2 \mathrm{~m}$. Il augmente (diminue) dans le nord de la mer d'Irlande, la partie méridionale de la mer du Nord et le long des côtes allemandes (Manche occidentale). Ces variations peuvent localement représenter jusqu'à $15 \%$ de la remontée du niveau marin. Dans le cas où le modèle est paramétré pour laisser l'eau se propager à terre (absence des défenses côtières), cette relation de proportionnalité s'altère, en particulier le long des côtes allemandes, tandis que d'autres zones sont peu sensibles à cet effet. Une estimation préliminaire des modifications des caractéristiques de marée d'ici 2100 est réalisée selon un scénario de remontée du niveau marin non-uniforme plausible, fondé sur le scénario RCP4.5, montrant que, localement, les modifications de la marée sont sensibles à la non-uniformité spatiale de la remontée du niveau marin. L'effet induit sur les courants de marée et le transport de sédiments est aussi discuté.

\section{Mots-clés :}

Marée, Plateau continental, Défenses côtières, Flux de sédiments, Modélisation, Projections.

\section{Introduction}

Les plaines côtières font partie des zones où la probabilité de submersion augmentera probablement en réponse au changement climatique et à la remontée du niveau moyen 


\section{Thème 1 - Hydrodynamique côtière}

des mers (SLR). En théorie, cette remontée a un effet direct sur le niveau d'eau instantanée, mais aussi un effet indirect entre autres sur la marée. A la fois à l'échelle globale et sur le plateau européen, l'analyse des données marégraphiques sur le dernier siècle montre des changements dans les caractéristiques des composantes primaires (M2, S2, K1, O1) de la marée (WOODWORTH, 2010). Si de nombreux phénomènes peuvent contribuer à ces changements (dragages, constructions de port, ...), en plus de la remontée du niveau marin, plusieurs études se sont concentrées sur l'effet de la remontée du niveau marin sur la marée du plateau ouest européen, et en particulier sur l'onde M2 (PICKERING et al., 2012), en considérant une remontée du niveau marin uniforme. D'après ces travaux, l'amplitude de l'onde M2 varie de $-15 \%$ à $15 \%$ de la remontée du niveau marin, et dans certaines zones semble varier proportionnellement avec la remontée du niveau marin.

L'objectif de la présente communication est d'approfondir ces travaux en particulier en : (1) analysant les modifications du niveau de pleine-mer (et non d'une composante tidale), (2) étudiant l'effet d'une remontée non-uniforme, (3) discutant l'effet induit entre autre sur le potentiel de flux de sédiment. Tout d'abord, la méthode est décrite, ensuite les effets d'une remontée uniforme du niveau marin et d'un scénario régional sont étudiés. Suit une discussion portant sur l'influence des stratégies de défenses côtières sur la marée et les implications en termes de submersion et de modification potentielle des flux de sédiment. Les travaux présentés s'appuient sur l'étude de IDIER et al. (2017) et complètent cette dernière en particulier concernant l'effet induit sur le potentiel de flux de sédiment.

\section{Méthode : modèle hydrodynamique et scénarios}

Le modèle numérique MARS, basé sur les équations en eau peu profonde, est utilisé (LAZURE \& DUMAS, 2008) pour simuler l'effet de la remontée du niveau marin sur la marée. Le modèle couvre le plateau nord-ouest européen (figure 1a). La taille de maille est de $2 \mathrm{~km}$. Les conditions aux limites sont générées à partir du modèle de marée global FES2004 (LYARD et al., 2006 ; 14 composantes : Mf, Mm, Msqm, Mtm, O1, P1, Q1, K1, M2, K2, 2N2, N2, S2, M4). Ce modèle a été validé en terme de marée à l'échelle de la zone d'étude (IDIER et al., 2017).

Dans ce qui suit, nous analysons les effets de SLR sur les marées, et plus précisément sur le niveau maximal de pleine-mer $\left(\xi_{\max }\right)$ par rapport au niveau moyen pour une année de type 2009. Dans un premier temps, on considère des scénarios sans submersion (appelés "Sc1"), i.e. où les mailles à terre ne peuvent pas être inondées, et on étudie l'effet de remontées du niveau marin spatialement uniformes de SLR=-0.25 m SLR=+5 $\mathrm{m}$. Ces scénarios incluent le scénario de contrôle correspondant à la situation actuelle $(\mathrm{SLR}=0 \mathrm{~m})$, ainsi que des scénarii idéalisés qui correspondent à des valeurs plausibles pour des horizons plus ou moins lointains dans la passé ou le futur. Le scénario $-0.25 \mathrm{~m}$ peut être considéré comme une limite basse du niveau marin de l'ère préindustrielle 


\section{XVèmes Journées Nationales Génie Côtier - Génie Civil \\ La Rochelle, 29 au 31 mai 2018}

(MITROVICA et al., 2015), tandis que la remontée probable pour un scénario de forte émission est de +0.5 à +1 m en 2100 ( $5^{\text {ième }}$ rapport de l'IPCC) - ce qui n'exclue pas des valeurs plus fortes -. Enfin, cette remontée atteindra probablement plusieurs mètres en 2200 (KOPP et al., 2014).


Figure 1. (a) Domaine de calcul du modèle hydrodynamique et localisation des jauges de validation du modèle. (b) Niveau de pleine mer maximal ( $\left.\xi_{\max }\right)$ pour le scenario de contrôle $(S L R=0 \mathrm{~m})$.

\section{Marées et remontée du niveau marin}

\subsection{Remontée uniforme}

La figure 2 illustre, pour une vive-eau moyenne, l'influence de la remontée du niveau moyen (SLR) sur la marée, pour plusieurs jauges : en fonction de la localisation, on observe des modifications de l'amplitude (augmentation ou diminution), de la phase temporelle (Scheveningen), voire de la forme de la courbe de marée (La Rochelle). L'analyse qui suit se concentre sur les modifications du plus haut niveau de pleine-mer $\left(\xi_{\max }\right)$. En chaque cellule du modèle, la différence $\Delta \xi_{\max }$ entre le niveau de pleine-mer (relatif du niveau moyen) atteint sur une année type 2009 pour une remontée du niveau marin SLR $\left(\xi_{\max }(\mathrm{SLR})\right)$ et le maximum atteint pour le scénario de contrôle $\left(\xi_{\max }(\mathrm{SLR}=0)\right.$, figure 3$)$. Pour comparer les figures spatiales des modifications du niveau de marée induites, nous étudions le ratio $\Delta \xi_{\max } / \mathrm{SLR}$. Ce ratio $\Delta \xi_{\max } / \mathrm{SLR}$ varie de $-15 \%$ à $+15 \%$ de SLR (figure 3). Les diminutions (augmentations) les plus fortes sont observées en Manche occidentale (sud de la mer du nord, Manche orientale, mer d'Irlande). Par exemple, la baie du Mont Saint-Michel montre une diminution de $15 \mathrm{~cm}$ pour une remontée du niveau marin de $+1 \mathrm{~m}$. Sur la majeure partie du domaine, tant que SLR $\leq+2 \mathrm{~m}, \Delta \xi_{\max } / \mathrm{SLR}$ a toujours le même signe et approximativement la même 


\section{Thème 1 - Hydrodynamique côtière}

valeur, i.e. le niveau de pleine-mer maximale varie proportionnellement à SLR. La figure 4 (scénario "Sc1") montre l'évolution de $\Delta \xi_{\max }$ en fonction de la remontée du niveau marin, en 3 points localisés respectivement en manche occidentale, mer d'Irlande et mer des Wadden, des zones représentatives des évolutions rencontrées (augmentation, diminution). L'approximation que $\xi_{\max }$ varie proportionnellement avec SLR tant que SLR $\leq+2 \mathrm{~m}$ apparait comme une approximation imparfaite mais raisonnable. L'analyse statistique réalisée par IDIER et al. (2017) confirme que les modifications du niveau de pleine mer maximal sont proportionnelles à SLR dans la majeure partie domaine tant que SLR $\leq+2 \mathrm{~m}$. Les modifications de la marée résultent en fait de processus locaux (ex: réduction du frottement au fond induite par l'augmentation totale de hauteur d'eau) et non locaux (ex: modification des propriétés de résonance du plateau), ce qui explique entre autres l'existence de zones répondant différemment à la remontée du niveau marin. Par exemple, les modifications en Manche résulteraient de la compétition entre (a) la réduction du frottement au fond et (b) les modifications de propriétés de résonance. Pour une analyse plus détaillée des mécanismes, voir (IDIER et al., 2017).
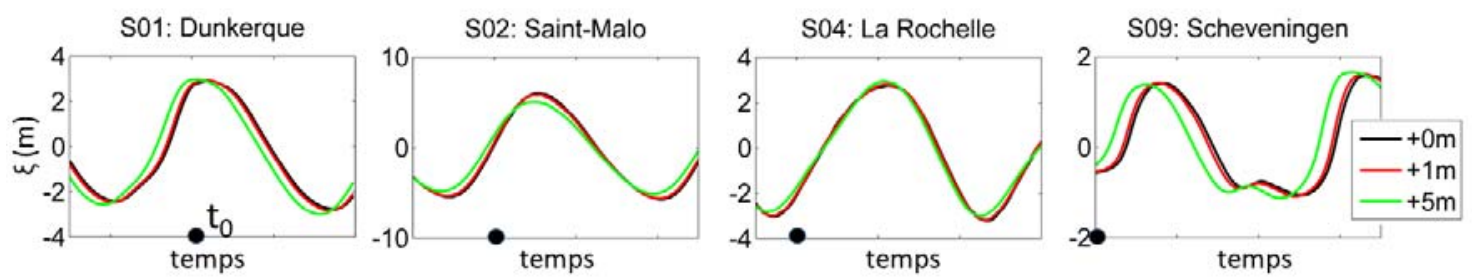

Figure 2. Courbes de marée pour les jauges S01, S02, S04, S09 (figure 1a) pour une vive-eau moyenne (équivalente à un forçage de marée se produisant le 12-13 janvier 2009 - le point noir correspond au 13 janvier 00h).
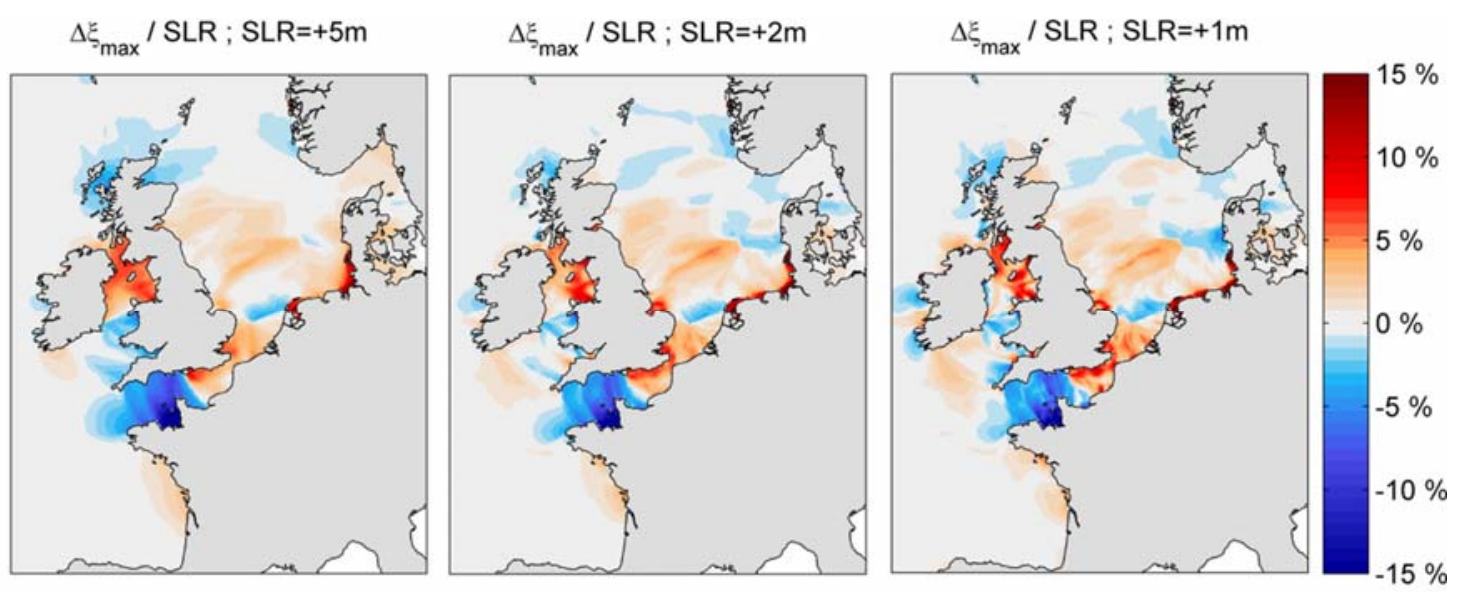

Figure 3. Changements relatifs du niveau de pleine mer maximal ( $\left.\triangle \xi_{\max } / S L R\right)$. 


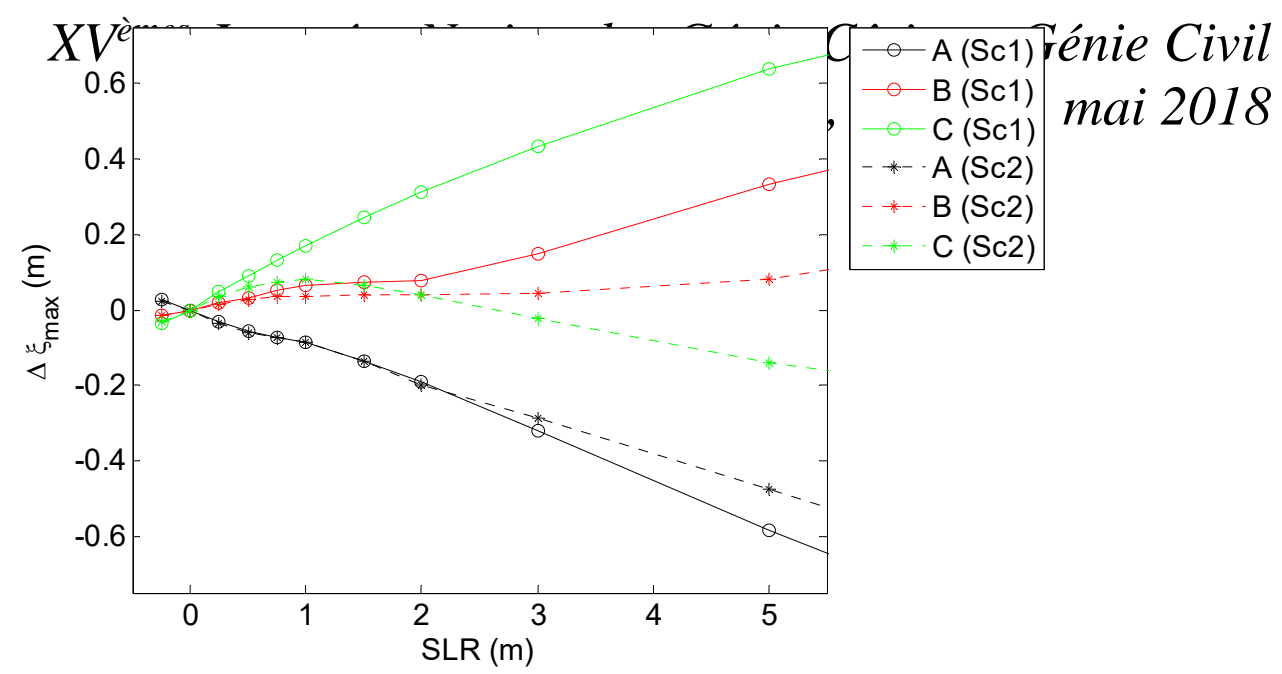

Figure 4. $\Delta \xi_{\max }$ en fonction de la remontée du niveau marin pour les points $A, B$ et $C$

(figure 1a) pour les scenarios avec (Sc2) et sans ( $S c 1)$ submersion autorisée.

\subsection{Variabilité régionale de la remontée du niveau des mers}

Les résultats ci-dessus sont basés sur l'hypothèse d'une remontée uniforme du niveau moyen des mers. Cependant, la remontée du niveau marin future présente des disparités régionales, induites par le réchauffement non-uniforme de l'océan et des déformations de la terre solide. Nous analysons ici dans quelle mesure les modifications de marée relatives au niveau marin induites par une remontée uniforme ou non-uniforme diffèreraient de manière significative en considérant une remontée non-uniforme synthétique idéalisée, fondée sur la projection régionale médiane du scénario RCP4.5 de SLANGEN et al. (2014) (figure 5a), qui implique une élévation globale de $0.5 \mathrm{~m}$ en 2100. La figure $5 \mathrm{~b}$ montre les modifications induites sur le niveau de pleine mer relatif. Pour estimer plus quantitativement l'effet de la non-uniformité de la remontée du niveau marin, la différence relative $(\Delta 1-\Delta 2) / \Delta 2$ est calculée, avec $\Delta 1=\left(\Delta \xi_{\max } / \mathrm{SLR}\right)$ les modifications pour le scenario scénario non-uniforme et $\Delta 2=\left(\Delta \xi_{\max } / \mathrm{SLR}\right)$ celles pour le scenario uniforme $\mathrm{SLR}=0.5 \mathrm{~m}$ (scénario le plus proche de la valeur moyenne du scénario non uniforme). Sur $92 \%$ du domaine, les différences sont inférieures à $1 \%$ (figure $5 \mathrm{c}$ ). Les plus fortes différences atteignent 10\%, principalement en mer d'Irlande, canal de Bristol et dans certaines baies. Les résultats obtenus pour ce scénario nonuniforme, fondé sur les connaissances existantes de la variabilité régionale future de la remontée du niveau marin, constituent une première évaluation quantitative des modifications de la marée (niveau de pleine mer) pour l'horizon 2081-2100 et un scénario RCP4.5. Ils montrent aussi que prendre en compte cette variabilité spatiale pourrait être utile pour réduire les incertitudes dans les estimations des modifications futures de la marée dans certaines zones. 


\section{Thème 1 - Hydrodynamique côtière}

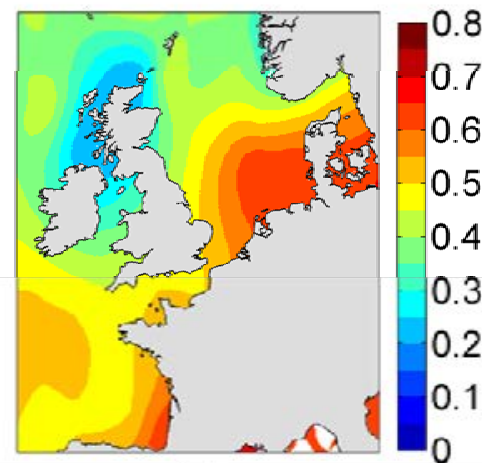

(a)

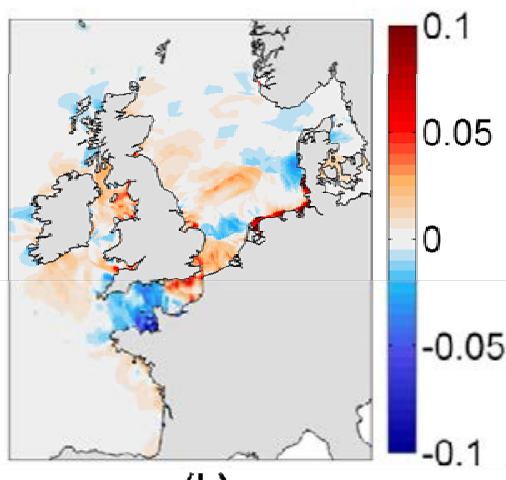

(b)

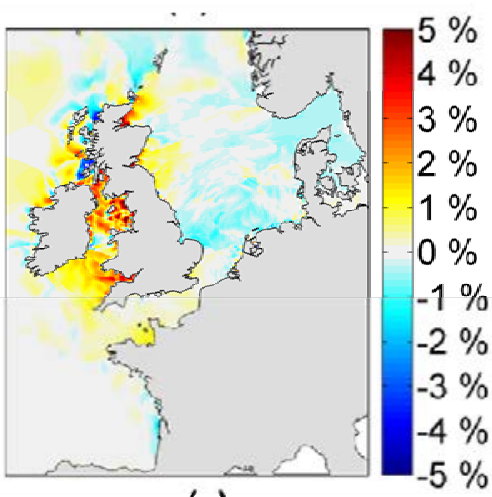

(c)

Figure 5. (a) Remontée du niveau marin non-uniforme $(m)$; (b) changements absolus $\Delta \xi_{\max }(\mathrm{m})$; (c) différence relative $(\Delta 1-\Delta 2) / \Delta 2(\%)$ avec $\Delta 1=\left(\Delta \xi_{\max } / S L R\right)(\%)$ pour le scénario non-uniforme et $\Delta 2=\left(\Delta \xi_{\max } / S L R\right)$ pour $S L R=0.5 \mathrm{~m}$.

\section{Discussion}

\subsection{Effet des défenses côtières}

Des simulations supplémentaires ont été réalisées en autorisant la submersion à terre (scénario Sc2, figure non montrée, voir IDIER et al., 2017). Dans 57\% du domaine, les changements relatifs $\left(\Delta \xi_{\max } / \mathrm{SLR}\right)$ obtenus ont le même signe que ceux pour le scénario Sc1 (cellules à terre non submersibles). Toutefois, dans certaines zones où $\xi_{\max }$ augmentait, une diminution est maintenant observée (ex., baie allemande, côtes néerlandaises). La figure 4 illustre ces comportements différents : dans certaines zones la relation de proportionnalité avec la remontée du niveau marin est conservée, tandis que d'autres ont un comportement très diffèrent (ex : point $\mathrm{C}$, mer de Wadden), par rapport au cas où la submersion n'est pas autorisée. Ainsi, au point $\mathrm{C}$, on observe d'abord une croissance de $\Delta \xi_{\max }$ jusqu'à $\mathrm{SLR}=+2 \mathrm{~m}$, puis une décroissance jusqu'à des valeurs négatives pour SLR supérieur à $+3 \mathrm{~m}$. On peut noter qu'une part significative des côtes néerlandaises et allemandes est sujette à ce comportement, probablement en raison de l'extension considérable de zones basses. Dans ces zones, les marées futures pourraient donc être fortement influencées par les stratégies de défenses côtières.

\subsection{Implications : submersion, courants et flux de sédiments}

La majeure partie des marégraphes le long des côtes mondiales (hors régions polaires) montre une hausse des niveaux d'eau extrêmes cohérente avec la remontée du niveau marin moyen (WOODWORTH et al., 2011). Les niveaux d'eau totaux futurs résulteront de la somme de la remontée du niveau marin relative, qui inclut les mouvements verticaux du sol, la marée, les surcotes et les composantes d'interactions. La remontée du niveau marin induira des modifications des niveaux de marée (localement jusqu'à 15\% de SLR), mais aussi les surcotes, directement ou indirectement via les processus d'interaction marée-surcote (qui peuvent atteindre plusieurs dizaines de centimètres, IDIER et al., 2012). Or, les différences de niveaux d'eau extrêmes entre une période de retour de 100 ans et 1000 ans ne sont généralement pas plus grandes que 


\section{XVèmes Journées Nationales Génie Côtier - Génie Civil \\ La Rochelle, 29 au 31 mai 2018}

quelques dizaines de centimètres. Ainsi, une modulation de 10 à $15 \%$ de la remontée du niveau marin par modification de la marée n'est pas négligeable en termes de prévention des risques et adaptation.

Les travaux présentés ci-dessus s'appuient sur de nombreuses hypothèses, comme par exemple une topo-bathymétrie inchangée. En dehors des zones de déferlement des vagues, on peut penser que cette hypothèse n'est toutefois pas aberrante, au regard des échelles de temps considérées (ici dizaines à centaine d'années). En gardant en tête cette hypothèse, des indicateurs d'effet de la remontée du niveau marin sur les courants et flux de sédiment sont estimés. Tout d'abord la vitesse $2 \mathrm{DH}$ maximum $\left(U_{\max }\right)$ est estimée sur une année type 2009, pour le scénario de $+1 \mathrm{~m}$ (et en considérant un scénario de type $\mathrm{Sc} 1)$. $U_{\max }$ diminue en Manche occidentale et augmente en mer d'Irlande et Mer du Nord. Les changements les plus forts atteignent $0.1 \mathrm{~m} / \mathrm{s}$. Concernant l'indicateur de dynamique sédimentaire, nous ne considérons que l'effet des courants de marée et estimons le volume de sédiments transporté (flux de sédiments cumulés x pas de temps) sur une année (2009) pour une taille de grain médiane de $0.25 \mathrm{~mm}$. Les flux de sédiments sont calculés en utilisant la formule de VAN RIJN (1989). Cet indicateur montre une forte diminution (10\%) en Manche occidentale (variation maximale de $10^{7}$ $\mathrm{kg} / \mathrm{m}$ ). Tout chose égale par ailleurs (ex : bathymétrie), localement, la remontée du niveau marin pourrait donc contribuer à modifier de manière significative le potentiel de dynamique sédimentaire induite par les courants de marée, en particulier en Manche.


Figure 6. Pour l'année 2009 et $S L R=0$ (a,c) et $S L R=+1 \mathrm{~m}(b, d)$ : (a) courant maximal $U_{\max }$, (b) modifications $\Delta U_{\max }(\mathrm{m} / \mathrm{s})$, (c) indicateur de volume de sédiments transportés IQ et (d) modifications de $\triangle I Q\left(\mathrm{~kg} \cdot \mathrm{m}^{-1}\right)$.

\section{Conclusions}

L'effet de la remontée du niveau marin a été étudié par modélisation. Ces travaux montrent que la remontée du niveau marin, mais aussi les stratégies de défenses côtières, ont un effet significatif sur les caractéristiques de marée dans des zones comme la Manche ou les côtes allemandes. La non-uniformité de la remontée du niveau marin peut aussi jouer localement. Ces modifications ne sont pas négligeables en termes de prévention des risques de submersion. Elles contribueront vraisemblablement à modifier aussi la dynamique sédimentaire, principalement en Manche. 


\section{Thème 1 - Hydrodynamique côtière}

\section{Références bibliographiques}

IDIER D., DUMAS F., MULLER H. (2012). Tide-surge interaction in the English Channel. Nat. Hazards Earth Syst. Sci., Vol. 12, pp 3709-3718. https://doi.org/10.5194/nhess$\underline{12-3709-2012}$

IDIER D., PARIS F., LE COZANNET G., BOULAHYA F., DUMAS F. (2017) Sealevel rise impacts on the tides of the European Shelf, Continental Shelf Research. https://doi.org/10.1016/j.csr.2017.01.007

KOPP R.E., HORTON R.M., LITTLE C.M., MITROVICA J.X., OPPENHEIMER M., RASMUSSEN D.J., STRAUSS B.H., TEBALDI C. (2014). Probabilistic 21st and 22nd century sea-level projections at a global network of tide-gauge sites. Earth's Future, Vol 2, pp 383-406. https://doi.org/10.1002/2014EF000239

LAZURE P., DUMAS F. (2008). An external-internal mode coupling for a 3D hydrodynamical model for applications at regional scale (MARS). Advances in Water Resources, Vol 31, pp 233-250. https://doi.org/10.1016/j.advwatres.2007.06.010

LYARD F., LEFEVRE F., LETELLIER T., FRANCIS O. (2006). Modelling the global ocean tides: modern insights from FES2004. Ocean Dynamics, Vol. 56(5-6), pp 394-415. https://doi.org/10.1007/s10236-006-0086-x

MITROVICA J.X., HAY C.C., MORROW E., KOPP R.E., DUMBERRY M., STANLEY S. (2015). Reconciling past changes in Earth's rotation with 20th century global sea-level rise: Resolving Munk’s enigma. Sci. Adv., Vol. 1, n 11, pp 1-6. https://doi.org/10.1126/sciadv.1500679

PICKERING M.D., WELLS N.C., HORSBURGH K.J., GREEN J.A.M. (2012). The impact of future sea-level rise on the European Shelf tides. Continental Shelf Research, Vol 35, pp 1-15. https://doi.org/10.1016/i.csr.2011.11.011

SLANGEN A.B.A., CARSON M., KATSMAN C.A., VAN DE WAL R.S.W., KÖHL A., VERMEERSEN L.L.A., STAMMER D. (2014). Projecting twenty-first century regional sea-level changes. Climatic change, Vol 124(1-2), pp 317-332. https://doi.org/10.1007/s10584-014-1080-9

VAN RIJN L.C. (1989). Handbook of sediment transport by currents and waves. Report H461, WL|Delft Hydraulics, The Netherlands.

WOODWORTH P.L. (2010). A survey of recent changes in the main components of the ocean tide. Continental Shelf Research, Vol 30, pp 1680-1691. https://doi.org/10.1016/j.csr.2010.07.002

WOODWORTH P.L., MENENDEZ M., GEHRELS W.R. (2011). Evidence for century-timescale acceleration in mean sea levels and for recent changes in extreme sea levels. Surv. Geophys., Vol 32(4-5), pp 603-618. https://doi.org/10.1007/s10712-011-9112-8 